\title{
Application of DELMIA on Maintainability Design of Aircraft
}

\author{
Shi Yongsheng \\ College of Aeronautical Engineering \\ Civil Aviation University of China \\ Tianjin, China, 300300
}

\author{
$\mathrm{Liu} \mathrm{Yu}$ \\ College of Aeronautical Engineering \\ Civil Aviation University of China \\ Tianjin, China, 300300
}

\begin{abstract}
The quality of the maintainability is the key to success of modern large aircraft, In this paper, the flow of interactive maintenance design (Design For Maintenance, DFM) is introduced based on the DELMIA software, Also the requirements of maintainability design. We established the models of Boeing 737-300 aircraft nose gear, and maintenance process of the gear is simulated. We also explored how to find and overcome the maintenance trouble in the design and maintenance aspects. From the perspective of accessibility and operability, maintainability analysis of landing gear maintenance is completed. Proof of using the DELMIA software, may realize the aircraft 'DFM' optimization.
\end{abstract}

Keywords-DELMIA, Design For Maintenance, maintainability, Maintenance Trouble

\section{INTRODUCTION}

Maintainability refers to the product which uses under the stipulation condition, within the stipulated time, according to the prescribed procedures and methods for maintenance, the ability to maintain or to restore of specified state. Maintainability conferred by the time of product design is an inherent attribute which makes maintenance simple, rapid and economic[1].In short, maintainability is not automatically generated, but is the characteristic which forms by the design. DFM can fundamentally improve aircraft maintenance characteristics in the phase of aircraft design.

The traditional aircraft maintainability design relied on physical prototypes or prototype, led maintenance-related research and development work for time-delay, designers and maintenance staff many problems such as poor communication and coordination. Using CAD technology for analyses of aircraft maintenance and human factors engineering have achieved significant results. In 1995, Lockheed Martin using CAD technology successfully solved the technical issues related to maintainability and human factors in F-16 project[2]. Afterwards, virtual simulating platforms DELMIA was used to simulate the processes of engines dismounting and weapons loading in JSF project of Lockheed Martin, which attained a lot of benefits in time and economic aspects[3]. In terms of improving product maintenance characteristics and production efficiency, shortening the development cycle, saving funds and so on, the introduction of virtual maintenance technology plays an important role. In this paper, on the base of CATIA and DELMIA software, combined with Boeing relevant manuals, we established the model of Boeing 737-300 aircraft nose gear, and simulated the maintenance process of the gear.
From the perspective of accessibility and operability, maintainability analyses of landing gear maintenance are completed.

\section{Maintainability Design ReQuirements}

Judging from the maintenance practices, maintainability design requirements mainly: simplifying products and maintenance operation, good accessibility, high standardization and interchangeability, and improving the measures to prevent the error, good maintenance security and testing, and so on. This paper from the perspectives of reachability and operability, we analyze maintainability of aircraft.

Accessibility refers to the maintenance or operation, the difficulty of reaching to different components of the products. Accessibility can be divided into: accessibility of maintenance places, to meet the different posture, position and other maintenance work; accessibility of machine external, maintenance of orifice location, size or method of setting the flap; the machine's internal accessibility, installation of the chassis parts, the layout of parts. Solve the reachability problem can be reached from the aspects of the visual, physical accessibility, adequate maneuvering space.

The operability obtained from the concrete operation (disassemblage, assembly, adjustment), makes maintenance easier and faster. The basic requirement of maintainability is the repair time must be short. Solve the operability problem can be reached from the aspects of meeting the body's physiological characteristics, system easy to be controlled.

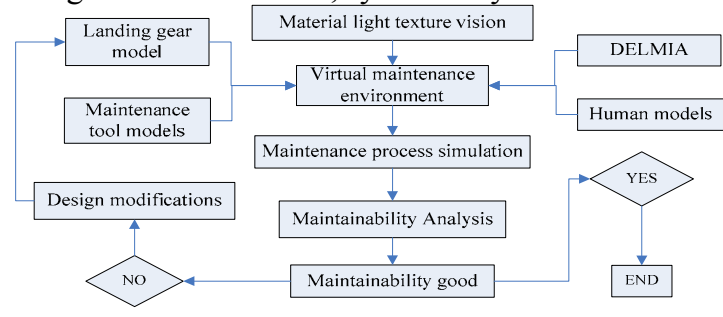

Figure 1. landing gear maintainability analysis

\section{Maintainability ANALysis Process}

CATIA and DELMIA (Digital Enterprise Lean Manufacturing Interactive Application) are the three dimensional design softwares which produced by France Dassault company. Their applications cover the aviation, aerospace, automotive and ship almost all digital manufacturing of machinery products. DELMIA divid digital 
manufactue into three different areas, namely: process planning; process refinement and validation; resource modeling and simulation. Resource modeling and simulation module include factory processes simulation, virtual reality and human factors engineering analysis.Based on this module, Landing gear maintenance process simulation and maintainability analysis can be achieved, Three-dimensional models(landing gear and maintenance tools) were established by the software of CATIA. The flow of maintainability analysis of landing gear is shown as following fig. 1

\section{A. The Establishment of 3D Models}

Digital modeling of landing gear parts and maintenance tools, reference to the Boeing Company information manuals (aircraft maintenance manuals, parts catalogs, standard construction manuals, repair manuals, etc), In conjunction with Boeing 737-300 landing gear with physical access to the parts information of geometry, texture, dimension and constraint. Application of 'mechanical design' subsystem of CATIA V5 software,we establish the models of Boeing 737300 nose landing gear and repair tools using the function of part design,surface design, components assembly. Constraint of landing gear parts as shown in fig.2, landing gear model as shown in the fig.3 (to facilitate the observation, landing gear wheel well transparent treatment here).

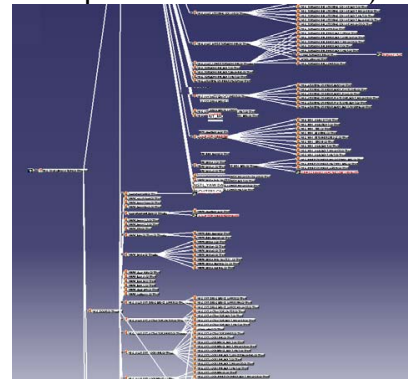

Figure 2. constraint of landing

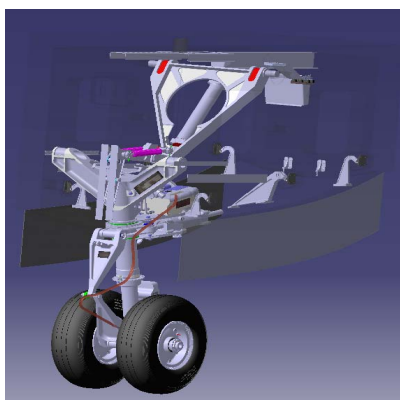

Figure 3. landing gear model

\section{B. Human Model}

The type of maintenance staff should consider maintenance mission and space constraints of maintenance operations position. Human model of DELMIA software contains extensive anthropometric data, including different races, different genders. We can also manually adjust the parameters of human model. For critical human dimension design limits, human body model can be established from the range of 0.01 to 99.99 percentile. Taking the actual maintenance into account, the maintenance staff needed to wear winter clothes or carrying other cumbersome tools, need more room to operate, so the maintenance staff model designed for the Asian race, the 95th percentile. Human model structure of DELMIA contains 68 joints, each joint has six degrees of freedom hinge. And includes the capture, placement, walking, and other basic movements, We can accurately simulate all kinds of maintenance actions through a combination of these movements and gestures editing.

\section{Maintenance Trouble}

Based on DELMIA software, you can implement virtual humans to maintenance the virtual product in virtual scene, and human factors analysis. We can import the models(landing gear model, human model, repair tool model)into DPM (Assembly Process Simulation) module for simulation. To find various maintenance obstacles in the areas of the maintenance of products, in order to improve the design and maintenance problems, and enhances the maintainability of the product.

Maintenance trouble is the events or status which make the products could not be repaired easily, quickly and economical. It can be divided into: Design Trouble; Technology Trouble; Operate Trouble based on the reason for trouble[4].

(1) Design Trouble can also be called the structural trouble, it is due to the unreasonable design of structure, the improper type of fasteners and other causes, resulting in troubles during the product repairing. For these problems, the information of equipment can be displayed on the computer based on DELMIA, also the overall layout of components, assembly relations, structure, materials, size of the flap and the way of open. We can find Design Trouble based on the fouction of analysis and detection(Spatial analyses, collision detection, interference detection) of DELMIA, and improve the design timely, Finally, solve the structural design of the maintenance trouble after Re-verification. Collision detection and interference detection are shown in fig.4.

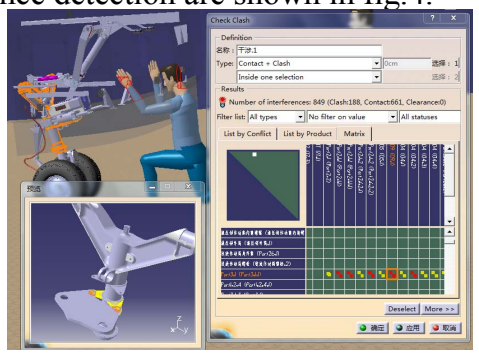

Figure 4. Collision detection and interference detection

(2) Technology Trouble occurred during maintenance due to improper formulation or implementation of maintenance process specification. Maintenance technology specifications often include processes of formulation, maintenance resource configuration. Early in the aircraft design, designers and maintenance personnel in the virtual environment to simulate the aircraft maintenance process based on DELMIA, and evaluate the maintainability. 
maintenance specification and training materials can be formulated by the provided data. Greatly save the time and solute Technology Trouble effectively.

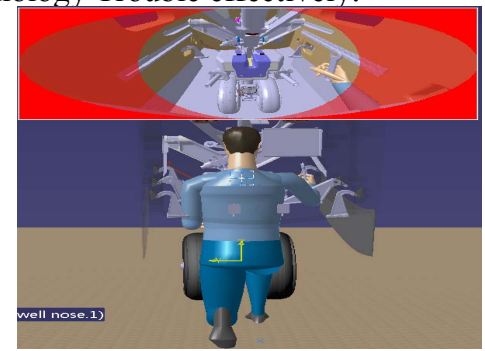

Figure 5. Analysis of visual accessibility

(3) Operate Trouble occurred during maintenance due to irregular maintenance conditions. Conditions here are generally divided into environmental conditions (maintenance of the facilities or places) that do not meet the requirements and maintenance operations due to obstacles caused by improper maintenance. We can build the virtual environment based on DELMIA, Also, human engineering analysis, such as visual analysis, biomechanical analysis, posture analysis. Analysis of visual accessibility of worker is shown in fig.5. The operation of the maintenance can intuitively reflect the accessibility and operability. And generate various analytical reports, used to improve the maintenance methods of operation. Make up for the human factors caused by Operate Trouble, Allows maintenance activities easy, Also, saving effort and time.

\section{Simulation Process}

Take replacing the nose landing gear wheels as an example, carries on maintainability analyze. The flow of removing wheels is shown in fig.6. This work can be completed by some people working together. From the perspective of accessibility and operability, we can analyses the maintainability and generate a variety of maintenance analysis report.

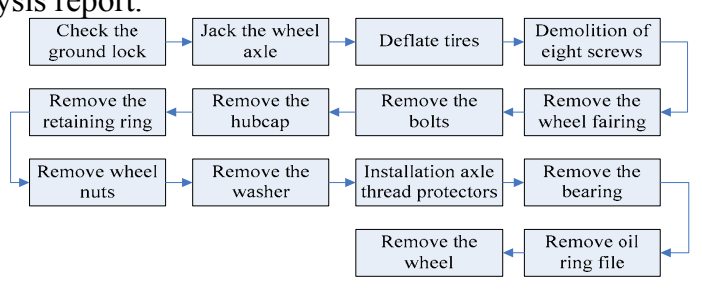

Figure 6. The flow of removing wheels

(1) Model import. the CATProduct format can directly import PPR(technology, products, resources) module. Other formats must be transformed through the middle form(IGS and STEP) to the CATProduct form. However, taking into account the model, affecting simulation effect, it is necessary to change the format, ignored some parts of topology relations so as to reduce file size. The models of maintenance objects (landing gear) were import to ProductList (products), the models of maintenance tools and maintenance workers were imported to ResourcesList (resources). Establish the maintenance scene.
(2) Maintenance tasks simulation. According to the actual process of replacing the wheel, we can determine the flow of maintenance process. We can organize and connect all Process by PERT Chart. Gantt Chart provides a visual resource applications, to view the relationship of all resources and maintenance action, and to adjust maintenance action time. The major maintenance process is shown in fig.7.
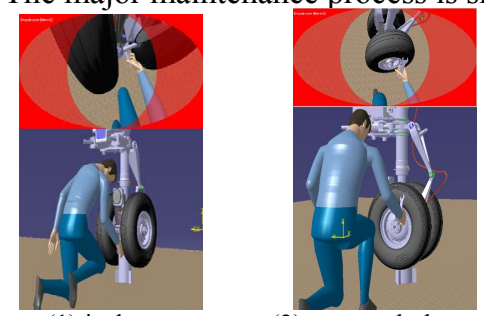

(1) jack to suppress (2) remove bolts
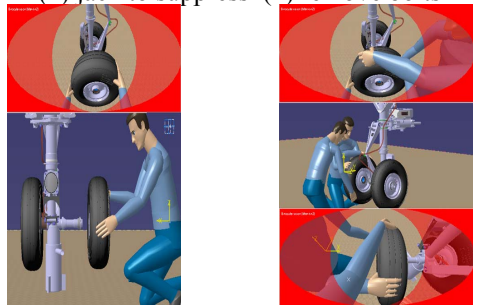

(3) remove wheel (4) install wheel

Figure 7. the major maintenance process

(3) Maintainability Analysis. From the perspective of accessibility, visual accessibility can be determined by the perspective of maintenance workers; physical accessibility can be observed by the function of Swept Volume, the movement of maintenance personnel and tools can be observed by swepting path of the volume. The paths for maintenance tools is shown in fig.8; We can analysis the accessibility of maneuvering space qualitative by the function of Distance And Band Analysis based on DELMIA.

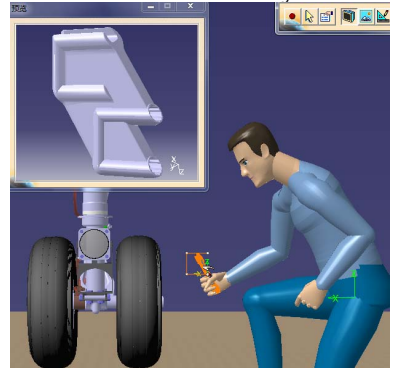

Figure 8. The paths for maintenance tools

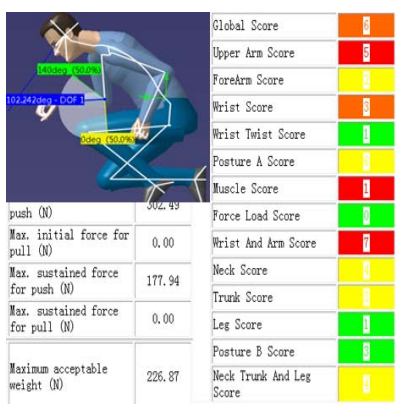


Figure 9. the analysis of actions

From the perspective of operability, maintenance action should be consistent with the body's physiological characteristics, namely, maintenance workers operate in a reasonable position to avoid fatigue; The body's physical limits should be considered; ensuring the maintenance of quality and efficiency, saving maintenance time. The posture analysis of maintenance personnel is provided by the module of Human Posture Analysis, can improve the gesture. In addition, DELMIA can generate a variety of analytical reports, such as push-pull analysis, rapid upper limb evaluation and analysis, bio-mechanical analysis and so on. the analysis of maintenance personnel actions is shown in figure 9. Obviously the scores of muscle and leg are low, and long time operation easy to cause wearily, proposed to adjust the position.

\section{SUMMERY}

The problems in terms of maintainability can be found and solved in early aircraft design by the application of DELMIA. the follow-up work related to maintenance also be supported. The optimization of DFM can be realized based on DELMIA.

\section{REFERENCES}

[1] S.Z.Tong, H.Q.Tong. "Maintenance and design of technologies," Beijing: China Standard Press, 2005: 1-12

[2] Kevin J Abshire,Mike K Barron.Virtual Maintenance Real-World Applications Within Virtual Enviroment[C].In:1998 Proceedings Annual Reliability and Maintain-ability Symposium,1998, 132-137

[3] "Gearing up for JSF assembly:simulating ERGOman at work," Tooling\&Production,2003, Vol.69, No.7, pp.20-23

[4] J.P.Hao, "Virtual Maintenance Simulation Theory and Technology," Beijing: National Defence Industry Press, 2007: 353-354 